\title{
Not there yet
}

\section{Although extraordinary scientific progress has been made, emerging challenges mean that the struggle against SARS-CoV-2 continues}

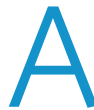

$s$ we enter the third year of the SARS-CoV-2 pandemic, Nature Immunology has commissioned a Series of Reviews and Perspectives that discuss the innate and adaptive aspects of the immune response to the virus, the possible mechanisms behind the large clinical variability in the response to infection, and considerations for vaccine and therapy strategies.

In a Review, Kanneganti and Diamond discuss the innate immune detection of SARS-CoV-2, including viral entry, pathogen recognition receptors and signaling pathways, the role of cytokine production and cell death, and viral immune evasion strategies. Enormous progress has been made over the past two years in understanding the viral replication process and identifying the molecules involved in detection and restriction. TLR1, TLR2, TLR4, TLR6, MDA5, LGP2, NLRP3, Ly6E and various IFIT proteins seem to be important players. It seems that SARS-CoV-2 can limit the production of type I and III interferon at several levels: at the stage of receptor sensing, induction, translation, expression, signaling or induction of IFN-stimulated genes (ISGs). The interferon response, in turn, seems to be crucial in setting the platform for the adaptive response.

The important link between the interferon response and the adaptive immune responses is also mentioned by Moss and colleagues in their Review, which discusses the characteristics of memory $\mathrm{T}$ cell responses and the molecular basis for cross-reactive recognition of SARS-CoV-2 and other human coronaviruses ( $\mathrm{HCoV})$. While the durability and breadth of the former may be the most critical factor in determining the outcome of this pandemic, the clinical importance of the latter is still unclear. On one hand, correlations between infection outcome and the presence of cross-reactive $\mathrm{T}$ cell responses have been documented, including in children and older individuals. On the other hand, $\mathrm{HCoV}$-specific $\mathrm{T}$ cells elicited by previous exposure often have low avidity against SARS-CoV-2 peptides, and it is possible that a de novo SARS-CoV-2-specific response is required for effective control. The relative contribution of pre-existing $\mathrm{HCoV}$-specific and newly recruited clones to the immune response and the clinical outcome of the infection, and how this contribution changes with age remains to be determined.

In their Review, which discusses the immune response to SARS-CoV-2 in children, Randolph and colleagues also point out that no definitive correlation between clinical outcomes and $\mathrm{T}$ cell epitope cross-reactivity has yet emerged. Several factors might explain the mild symptoms observed in children. There is evidence that children target different viral epitopes in comparison to adults; that they have lower expression of TMPRSS2 and ACE2, which control viral entry, and increased interferon responses and increased frequencies of $\mathrm{CD} 4^{+} \mathrm{T}$ cells that target non-structural viral proteins known to block the host interferon response; and that they develop less inflammation during infection. However, some children develop a broad post-infectious (which distinguishes it from severe COVID-19) hyperinflammatory complication referred to MIS-C, for which the risk factors remain unknown.

Similarly, the factors that lead or contribute to the post-infection syndrome known as long COVID are unknown. In their Review, Mehandru and Merad point out that it is important to distinguish between lingering symptoms of the acute disease, which seem to wane around six months after infection, and the development of new symptoms, sometimes in patients who had mild disease during the acute infection, and which persist for 24 months (for now) after infection. Unfortunately, owing to the almost complete lack of studies in this field, the authors can only extrapolate from observations in patients with severe acute infection and speculate on the factors that might contribute to long COVID, such as persistent increases in proinflammatory cytokines, persistence of the virus, triggered autoimmunity or tissue injury at levels that cannot be detected by clinical tests. They hypothesize that SARS-CoV-2 may fundamentally alter immune homeostasis mechanisms that modulate tissue inflammation. At the other end of the immune response spectrum, Spaan and colleagues discuss the genetic and immunological basis of inborn resistance to SARS-CoV-2.
All the Series authors discuss how these insights inform current and future therapeutic and vaccine strategies. Kanneganti and Diamond overview the various therapeutic approaches that target innate immunity in COVID-19, all with limited success. In a Review that will be published in an upcoming issue, Sekaly and colleagues point out that vaccines and adjuvants that modulate the intrinsic innate antiviral immunity should be less vulnerable to viral evolution and variant emergence, and that vaccine development must also consider contributing factors such as endemic co-infections, microbiota, antibiotics use, underlying conditions and age.

We need to know more, and quickly. Hospitals and institutes need to build the infrastructure required for easy access to clinical samples or patient cohorts and coordinate interactions between clinicians and researchers. Exquisite experimental design and interdisciplinary collaborations are required to enable broad, detailed and informative assessments of patient samples. Controls should include patients with relevant conditions, such as other viral infections, inflammatory diseases or autoimmune disorders, as well as asymptomatic patients. There is a need for better assessment of the immune response in asymptomatic patients and for in-depth immunological profiling of long COVID. As suggested by Mehandru and Merad, the latter should include repetitive measurements of pro-inflammatory cytokines, autoantibodies, viral or antigen persistence in stool samples, colon biopsies or other bodily fluids and in-depth profiling of immune subsets in blood and tissue biopsies. Viral persistence could also be assessed by testing whether antiviral therapies, including monoclonal antibody treatments, work against long COVID. It is essential that stable vaccines and effective treatments are developed. As the social and economic aspects of the pandemic continue to fuel the emergence of new virus variants, there is no easy way out. All we can do is tackle the challenges ahead.

Published online: 1 February 2022 https://doi.org/10.1038/s41590-022-01136-y 